Reduct i on of Turbul ent Transport wi th Zonal Fl ows Enhanced in Hel i cal Systens

\begin{tabular}{|l|l|}
\hline $\begin{array}{l}\text { jour nal or } \\
\text { publ i cat i on ti t l e }\end{array}$ & Physi cal Revi ew Let ter s \\
\hline vol une & Vol .100 \\
\hline page r ange & 195002-1 - 195002-4 \\
\hline year & 2008 05-01 \\
\hline URL & ht t p: //hdl . handl e. net /10655/2280 \\
\hline
\end{tabular}




\title{
Reduction of Turbulent Transport with Zonal Flows Enhanced in Helical Systems
}

\author{
T.-H. Watanabe, ${ }^{1,2}$ H. Sugama, ${ }^{1,2}$ and S. Ferrando-Margalet ${ }^{1}$ \\ ${ }^{1}$ National Institute for Fusion Science Toki, Gifu, 509-5292, Japan \\ ${ }^{2}$ The Graduate University for Advanced Studies (Sokendai), Toki, Gifu, 509-5292, Japan
}

(Received 12 February 2008; published 16 May 2008)

\begin{abstract}
Gyrokinetic Vlasov simulations of the ion temperature gradient turbulence are performed in order to investigate effects of helical magnetic configurations on turbulent transport and zonal flows. The obtained results confirm the theoretical prediction that helical configurations optimized for reducing neoclassical ripple transport can simultaneously reduce the turbulent transport with enhancing zonal-flow generation. Stationary zonal-flow structures accompanied with transport reduction are clearly identified by the simulation for the neoclassically optimized helical geometry. The generation of the stationary zonal flow explains a physical mechanism for causing the confinement improvement observed in the inwardshifted plasma in the Large Helical Device [O. Motojima et al., Nucl. Fusion 43, 1674 (2003)].
\end{abstract}

Magnetically confined toroidal plasmas involve two distinctive transport processes: that is, the neoclassical and turbulent diffusions. The neoclassical transport is strongly influenced by geometry of the toroidal field and is especially important for determining confinement properties in helical systems [1], where particles trapped in helical ripples cause significant radial particle and heat fluxes. The turbulent transport driven by microinstabilities [2] are widely observed in fusion plasma experiments while the $\boldsymbol{E} \times \boldsymbol{B}$ zonal flows have been investigated in numerous theoretical, numerical, and experimental studies as an attractive mechanism for regulating the turbulent transport [3-6]. So far, the two transport processes have been separately treated in conventional frameworks although it has been argued in recent theories that there exists a close relation between the neoclassical and anomalous transport through generation of zonal flows in helical systems [7-11].

Gyrokinetic theoretical studies of zonal flows driven by the ion temperature gradient (ITG) turbulence [7-9] show that a high-level zonal flow can be maintained for a longer time by reducing bounce-averaged radial drift velocity of helical-ripple-trapped particles. This means that optimization of the three-dimensional magnetic configuration for reducing the neoclassical ripple transport [12-14] simultaneously enhances the residual zonal flows which can lower the anomalous transport as well [7-11]. In fact, it is observed in the Large Helical Device (LHD) [15] experiments that the anomalous transport decreases in the inward-shifted plasma configuration optimized for reducing the neoclassical transport [16]. Thus, it is desired to validate the theoretical prediction of the transport reduction in the neoclassically optimized helical configurations by gyrokinetic simulations.

Preliminary simulations of the ITG turbulence and the zonal flows in helical systems were performed [17] by means of the gyrokinetic Vlasov simulation code GKV [18] and demonstrated stronger generation of zonal flows in a model configuration for the inward-shifted LHD plasma. However, the stationary zonal-flow structure effectively regulating the turbulent transport was not clearly observed, and the obtained $\chi_{i}$ in the inward-shifted case was higher than that in the standard one in contrast to the LHD experimental results. This is attributed to discrepancies in the linear ITG growth rates and the zonal-flow response between the numerical and experimental conditions.

In our recent study [9] on the linear ITG stability and the zonal-flow response relevant to the LHD experiments, we have employed more accurate confinement field models. Stabilizing effects of the smaller safety factor and the stronger magnetic shear associated with the inward plasma shift decrease the difference of the ITG mode growth rates between the two cases. Also, specified changes in the helical field components, the safety factor, and the aspect ratio clearly show that an initially given zonal flow keeps a higher level for a longer time in the inward-shifted configuration than that in the standard one. Both favorable changes in the zonal-flow response and the linear stability are expected to contribute to reduction of the ITG turbulent transport in the inward-shifted LHD configuration. Indeed, in this Letter, the nonlinear GKV simulation implemented with the relevant magnetic field parameters confirms generation of large zonal flows enough to reduce the ion heat transport in the inward-shifted plasma. Stationary zonalflow structures are clearly shown in the present simulation for the helical system with the neoclassical optimization. The obtained results agree with our theoretical analysis and are consistent with observation of better confinement in the inward-shifted LHD plasma [16].

The nonlinear gyrokinetic equation for the perturbed ion gyrocenter distribution function in the low- $\beta$ electrostatic limit is numerically solved by the GKV code [18] as a partial differential equation defined on the fivedimensional phase space. We introduce a collision model given by the gyrophase average of the Lenard-Bernstein 
collision operator [19]. The quasineutrality condition and the electron response of $\delta n_{e} / n_{0}=e(\phi-\langle\phi\rangle) / T_{e}$ are used for calculation of the electrostatic potential $\phi$, where $T_{e}$ and $\langle\cdots\rangle$ represent the electron temperature and the flux-surface average. In the GKV code, we employ the toroidal flux-tube coordinates $(x, y, z)$, where $y$ and $z$ are defined in terms of the poloidal angle $\theta$, toroidal angle $\zeta$, and the safety factor $q$ as $y=\left(r_{0} / q_{0}\right)[q(r) \theta-\zeta]$ and $z=$ $\theta$, respectively [20]. The radial coordinate $x$ is given by the average minor radius $r$, such that $x=r-r_{0}\left(\ll r_{0}\right)$. Here, $r$ is defined by $\Psi_{t}=\pi B_{0} r^{2}$, and $\Psi_{t}$ means the toroidal flux. The minor radius, at which local background parameters are evaluated, is represented by $r_{0}$. Then, $q_{0}=q\left(r_{0}\right)$. We also assume constant background density and temperature gradients with scale lengths of $L_{n}$ and $L_{T}$ as well as the constant magnetic shear parameter, $\hat{s}=\left(r_{0} / q_{0}\right) \times$ $(d q / d r)_{r=r_{0}}$. The parallel velocity $v_{\|}$and the magnetic moment $\mu$ are taken as the velocity-space coordinates.

Here, we consider large-aspect-ratio helical systems with

$$
\begin{aligned}
B= & B_{0}\left\{1-\epsilon_{00}(r)-\epsilon_{t}(r) \cos z\right. \\
& \left.-\sum_{l=L+1}^{l=L-1} \epsilon_{l}(r) \cos \left[\left(l-M q_{0}\right) z-M \alpha\right]\right\},
\end{aligned}
$$

where $L$ and $M$ denote the poloidal and toroidal period numbers of the main component of the helical field. For the LHD, $L=2$ and $M=10$. The main helical field is represented by $\epsilon_{L}$, while the sideband components and the averaged normal curvature are given by $\epsilon_{L-1}, \epsilon_{L+1}$, and $\epsilon_{00}^{\prime}=d \epsilon_{00} / d r$, respectively. The confinement field parameters are chosen so as to reproduce the inward-shifted and standard LHD configurations (for each value, see cases I-B and S-B given in Table I of Ref. [9]). We also set the field-line label $\alpha$ to be constant $(\alpha=0)$ in Eq. (1) because of negligibly weak $\alpha$ dependence of the linear ITG instability [21] and the zonal-flow response [7,8] in the large $M$ limit. In order to mainly examine magneticconfiguration effects, the same local plasma parameters are used for the two cases, such that $\eta_{i} \equiv L_{n} / L_{T i}=3$, $L_{n} / R_{0}=0.3$, and $T_{e} / T_{i}=1$. The collision frequency is given by $\nu=0.002 v_{t i} / L_{n}$ for both cases, where $R_{0}, T_{i}$, and $v_{t i}$ denote the major radius, the ion temperature, and the ion thermal speed, respectively.

The ITG turbulence simulations for helical systems with a high toroidal period number $M \gg 1$ demand fine numerical resolution along the field line. In addition, motion of helical-ripple-trapped particles causes complicated fine structures of the distribution function in the phase space $[8,9]$. Thus, we employ a huge number of grid points over $5 \times 10^{10}$ in total [that is, $128 \times 128 \times 768 \times 128 \times 48$ for the inward-shifted case or $128 \times 128 \times 512 \times 128 \times 48$ for the standard case in the $\left(x, y, z, v_{\|}, \mu\right)$ coordinates] so that the entropy balance [18] is accurately satisfied within an error less than $7 \%$. The minimum poloidal wave number is given by $k_{y, \min } \rho_{i}=0.0463$ (the system length of $L_{y} \equiv$ $2 \pi / k_{y, \min }=135.7 \rho_{i}$ covers $1 / 6$ of the whole flux surface) while the minimum radial wave number is $k_{x, \min } \rho_{i}=$ $0.140\left(L_{x} \equiv 2 \pi / k_{x, \min }=44.9 \rho_{i}\right)$ for the inward-shifted case, and $k_{x, \min } \rho_{i}=0.124\left(L_{x} \equiv 2 \pi / k_{x, \min }=50.7 \rho_{i}\right)$ for the standard case, where $\rho_{i}$ means the thermal ion gyroradius. The small difference in $k_{x \text {, min }}$ stems from change of the magnetic shear parameter $\hat{s}$ (that is, $\hat{s}=-0.96$ and -0.85 for the inward-shifted and standard configuration cases, respectively [9]), but has no essential influence on the resultant transport. Further details of the GKV simulation model are found in Refs. $[9,17,18]$.

The time history of the flux-surface-averaged potential obtained by the linear GKV simulation of the collisionless zonal-flow damping is shown in Fig. 1, where, for comparisons with the ITG turbulence simulation results shown below, the radial wave numbers of $k_{x} \rho_{i}=2 k_{x, \min } \rho_{i}=$ 0.280 and 0.248 are used for the inward-shifted and the standard cases, respectively. Slower decay and higher residual level of the zonal-flow amplitude are obviously found in the inward-shifted configuration. This improved zonal-flow response for the neoclassically optimized geometry agrees with the theoretical prediction. For the case of Fig. 1, the enhancement of the residual level by about $50 \%$ is comparable with the increase by about $30 \%$ which is derived from the theoretical formulas $[7,8]$.

The ion thermal diffusivity $\chi_{i}$ obtained by the GKV simulation of the ITG turbulence is shown in Fig. 2 as a function of time $t$ for the inward-shifted (solid line) and standard (dashed line) configurations. It is found that, as expected from the linear stability analysis [9], $\chi_{i}$ in the linear growth stage of the instability $\left(t<40 L_{n} / v_{t i}\right)$ grows slightly faster for the inward-shifted configuration than for the standard case, and that the peak value of $\chi_{i}$ for the former case is larger than that for the latter case. In the saturated ITG turbulence $\left(t>60 L_{n} / v_{t i}\right)$, however, the

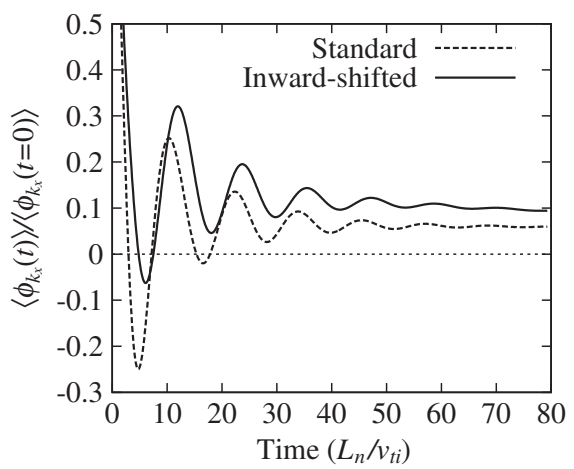

FIG. 1. Time history of the flux-surface averaged potential obtained from the linear GKV simulation of the collisionless zonal-flow damping for the inward-shifted (solid line with the radial wave number $k_{x} \rho_{i}=0.280$ ) and the standard (dashed line with $\left.k_{x} \rho_{i}=0.248\right)$ model configurations of the Large Helical Device (LHD) experiment. 


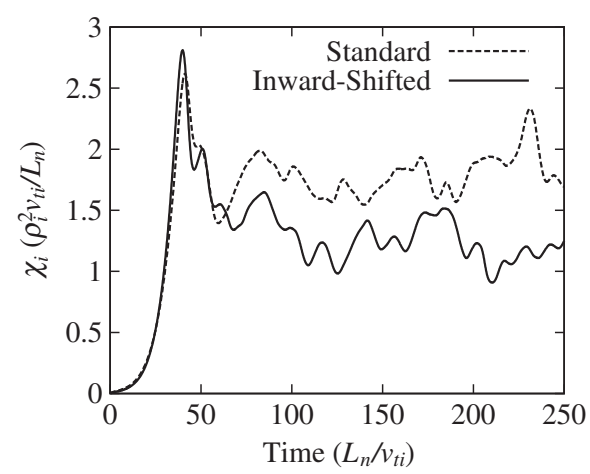

FIG. 2. Time history of the ion heat conductivity $\chi_{i}$ resulted from the GKV simulation of the ion temperature gradient (ITG) turbulent transport for the inward-shifted (solid line) and the standard (dashed line) LHD configurations.

time-averaged ion thermal diffusivity $\chi_{i} \approx 1.27 \rho_{i}^{2} v_{t i} / L_{n}$ for the inward-shifted plasma is about $30 \%$ smaller than the time-averaged value of $\chi_{i} \approx 1.78 \rho_{i}^{2} v_{t i} / L_{n}$ for the standard one. The evident transport reduction in the inward-shifted case is attributed to a larger amplitude of zonal flows generated by turbulence as shown below, and is in contrast to the previous simulation result for the simpler model cases in Ref. [17]. This is a consequence of the aforementioned slower decay and higher residual level of the zonal-flow response in the inward-shifted case as well as the reduction of the difference in the linear growth rates of the ITG instability between the two cases.

Color contours of the electrostatic potential $\phi$ in the nonlinear saturation phase at $t=120 L_{n} / v_{t i}$ are shown in Fig. 3. The potential fluctuations are mapped on the innermost flux surface of the simulation box and the elliptic poloidal cross section by simultaneously plotting the color contours on the six equivalent flux-tube simulation domains. The ballooning-type mode structures found in the linear growth phase of the instability are destroyed by the self-generated $\boldsymbol{E} \times \boldsymbol{B}$ zonal flows in the later turbulent state as seen in the color figures. In the potential patterns
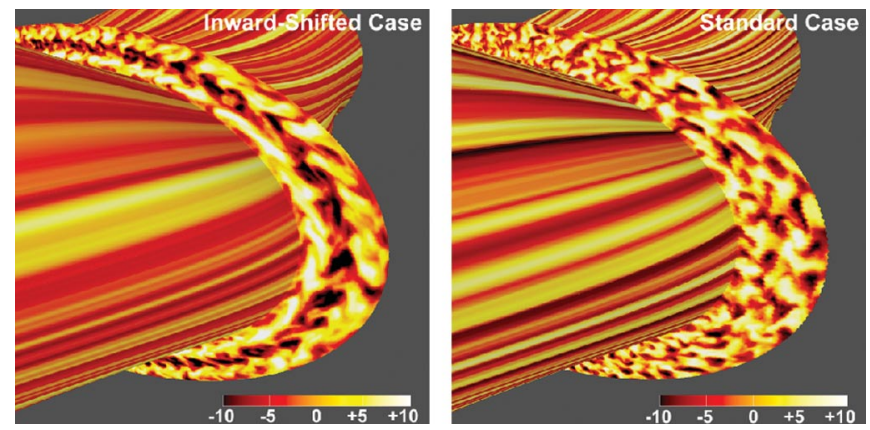

FIG. 3 (color). Color contours of the electrostatic potential $\phi$ of the zonal flow and the ITG turbulence obtained by the GKV simulation for the inward-shifted (left) and standard (right) LHD configurations at $t=120 L_{n} / v_{t i}$, where a part of the helical tori are zoomed in. Numbers below the color bars represent the values of the normalized potential $e \phi L_{n} / T_{e} \rho_{i}$. on the poloidal plane, the zonal-flow structures are more obviously identified in the inward-shifted configuration and cause the lower $\chi_{i}$ than for the standard one shown in Fig. 2.

Radial profiles of the flux-surface-averaged potential $e\left\langle\phi_{k_{y}=0}(x)\right\rangle L_{n} / T_{e} \rho_{i}$ shown in Fig. 4 indicate the stronger generation of stationary zonal flows in the inward-shifted case, where the potential data are time averaged from $t=$ 60 to $t=250 L_{n} / v_{t i}$. A sinusoidal mode structure dominates in the radial profile of the stationary zonal flows in the inward-shifted case. The peak value of $\left\langle\phi_{k_{y}=0}(x)\right\rangle \approx$ $4.5 T_{e} \rho_{i} / e L_{n}$ for the inward-shifted case is about 6 times larger than the largest amplitude of $\left\langle\phi_{k_{y}=0}(x)\right\rangle \approx$ $0.72 T_{e} \rho_{i} / e L_{n}$ for the standard one. Radial wave numbers $k_{x}$ for the dominant component of the stationary zonal flows are the same as those employed in the linear response analysis shown in Fig. 1. As expected in our previous works [7-9,17], the stronger zonal-flow generation in the inward-shifted configuration agrees with the improvement of the zonal-flow response by the neoclassical optimization.

The transport reduction associated with the zonal-flow generation is clearly recognized in Lissajous plots of time histories of the simulation data in Fig. 5, where the vertical axis measures the squared potential of the zonal flow, $\sum_{k_{x}}\left|e\left\langle\phi_{k_{x}, 0}\right\rangle L_{n} / T_{e} \rho_{i}\right|^{2}$. The horizontal axes in the left and right panels of Fig. 5 stand for the turbulent fluctuations, $\sum_{k_{x}, k_{y}}\left\langle\left|e \phi_{k_{x}, k_{y}} L_{n} / T_{e} \rho_{i}\right|^{2}\right\rangle$, and the transport coefficient, $\chi_{i} /\left(\rho_{i}^{2} v_{\mathrm{ti}} / L_{n}\right)$, respectively. In the left and right panels of Fig. 5, starting from the initial condition near the origin $(0,0)$, the data points move along the horizontal axis as the instability grows. In the nonlinear stage of the instability, the data points shift upward as the zonal flow is generated. One can see that the ion heat diffusivity $\chi_{i}$ is reduced when the zonal flow is enhanced, where the orbit of the data point moves in the upper-left direction of the figure. In the nonlinear saturation stage of the instability $\left(t>60 L_{n} / v_{t i}\right)$, the data point for the standard configuration (dashed line) stays in the region with higher transport and weaker zonal flows, while it is found in the region with

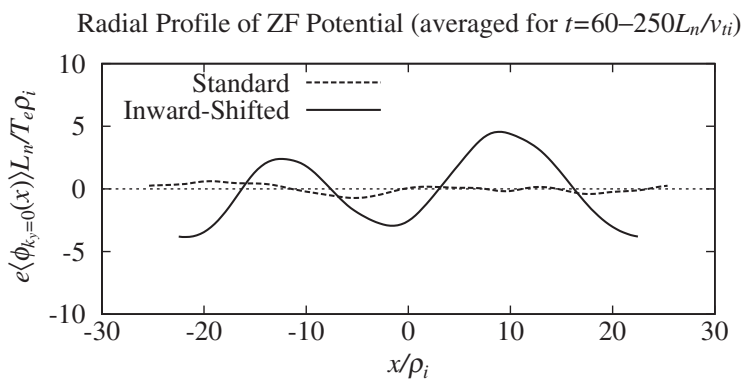

FIG. 4. Radial profiles of the zonal-flow (ZF) potential averaged from $t=60$ to $250 L_{n} / v_{t i}$. Solid and dashed curves represent the results obtained from the inward-shifted and standard cases, respectively. 

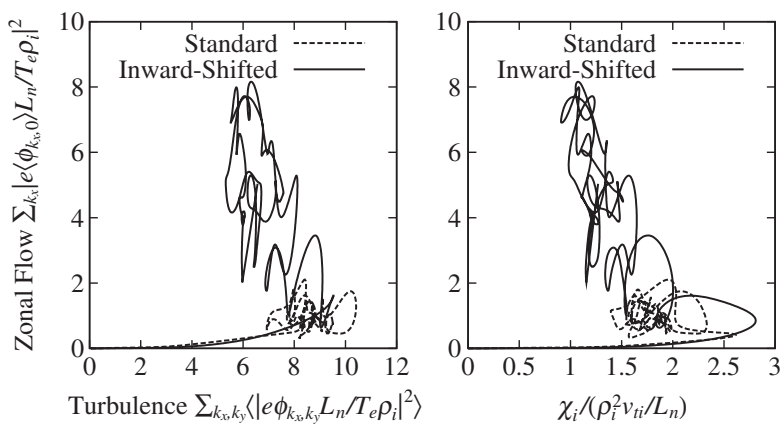

FIG. 5. Lissajous plots of the simulation results for the inward-shifted (solid lines) and standard (dashed lines) model cases. The vertical axis represents the squared potential of the zonal flow, $\sum_{k_{x}}\left|e\left\langle\phi_{k_{x}, 0}\right\rangle L_{n} / T_{e} \rho_{i}\right|^{2}$. The horizontal axes in left and right panels measure the turbulent fluctuations, $\sum_{k_{x}, k_{y}}\left\langle\left|e \phi_{k_{x}, k_{y}} L_{n} / T_{e} \rho_{i}\right|^{2}\right\rangle$, and the transport coefficient, $\chi_{i} /\left(\rho_{i}^{2} v_{\mathrm{ti}} / L_{n}\right)$, respectively.

lower transport and stronger zonal flow for the inwardshifted one. The Lissajous plots also manifest that the zonal flows for the inward-shifted case have much larger averaged amplitudes than those for the standard case. In the former case, one can see a correlation that both the turbulent energy and the ion heat diffusivity are decreased as the zonal flow amplitude increases.

The nonlinear gyrokinetic Vlasov simulations demonstrate that the ion thermal diffusivity $\chi_{i}$ driven by the ITG turbulence for the inward-shifted LHD plasma takes a lower time-averaged value in the steady turbulent state due to the stronger generation of stationary zonal flows. The obtained results clearly show that the neoclassical optimization leads to reduction of the anomalous transport through enhancement of the zonal flow $[7,8]$ and explains a probable physical mechanism of the confinement improvement found in the inward-shifted configurations of the LHD experiments. Extension of the present gyrokinetic simulation as well as more detailed comparison with experimental results is planned for future studies to investigate effects of the radial electric field driven by the neoclassical ripple transport, arbitrary helical geometry, the nonadiabatic electrons, and electromagnetic fluctuations.

This work is supported in part by grants-in-aid of the Ministry of Education, Culture, Sports, Science and
Technology (No. 16560727, No. 17360445, and No. 1705373), and in part by the National Institute for Fusion Science (NIFS) Collaborative Research Program (NIFS05KKMT001, NIFS06KTAT038, NIFS06KDAD006, and NIFS06KNXN060). Numerical simulations are carried out by use of the Earth Simulator under the support by Japan Agency for Marine-Earth Science and Technology, and by use of the Plasma Simulator and the LHD numerical analysis system at National Institute for Fusion Science.

[1] M. Wakatani, Stellarator and Heliotron Devices (Oxford University, New York, 1998), Chap. 7.

[2] W. Horton, Rev. Mod. Phys. 71, 735 (1999).

[3] M. N. Rosenbluth and F. L. Hinton, Phys. Rev. Lett. 80, 724 (1998).

[4] P. H. Diamond, S.-I. Itoh, K. Itoh, and T. S. Hahm, Plasma Phys. Controlled Fusion 47, R35 (2005).

[5] Z. Lin et al., Science 281, 1835 (1998).

[6] A. Fujisawa et al., Phys. Rev. Lett. 93, 165002 (2004).

[7] H. Sugama and T.-H. Watanabe, Phys. Rev. Lett. 94, 115001 (2005).

[8] H. Sugama and T.-H. Watanabe, Phys. Plasmas 13, 012501 (2006).

[9] S. Ferrando-Margalet, H. Sugama, and T.-H. Watanabe, Phys. Plasmas 14, 122505 (2007).

[10] H. E. Mynick, Phys. Plasmas 13, 058102 (2006).

[11] K. C. Shaing, Phys. Plasmas 12, 082508 (2005).

[12] S. Murakami et al., Nucl. Fusion 42, L19 (2002).

[13] M. Yokoyama and K. Y. Watanabe, Nucl. Fusion 45, 1600 (2005).

[14] D. A. Spong, Phys. Plasmas 12, 056114 (2005).

[15] O. Motojima et al., Nucl. Fusion 43, 1674 (2003).

[16] H. Yamada et al., Plasma Phys. Controlled Fusion 43, A55 (2001).

[17] T.-H. Watanabe, H. Sugama, and S. Ferrando-Margalet, Nucl. Fusion 47, 1383 (2007).

[18] T.-H. Watanabe and H. Sugama, Nucl. Fusion 46, 24 (2006).

[19] P. C. Clemmow and J.P. Dougherty, Electrodynamics of Particles and Plasmas (Addison-Wesley, Redwood City, 1969).

[20] M. A. Beer, S. C. Cowley, and G. W. Hammett, Phys. Plasmas 2, 2687 (1995).

[21] T. Kuroda, H. Sugama, R. Kanno, and M. Okamoto, J. Phys. Soc. Jpn. 69, 2485 (2000). 